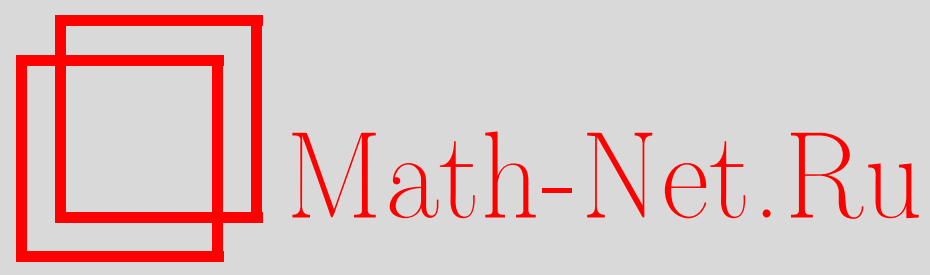

Е. А. Калита, О равенстве единице весовых норм преобразований Рисса, Матем. заметки, 2002, том 72, выпуск 6, 869882

DOI: https://doi.org/10.4213/mzm473

Использование Общероссийского математического портала Math-Net.Ru подразумевает, что вы прочитали и согласны с пользовательским соглашением http://www.mathnet.ru/rus/agreement

Параметры загрузки:

IP : 54.166 .219 .16

26 апреля 2023 г., 03:47:04

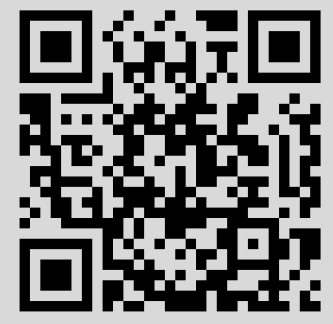




\section{O РАВЕНСТВЕ ЕДИНИЦЕ ВЕСОВЫХ НОРМ ПРЕОБРАЗОВАНИЙ РИССА}

\section{Е.А. Калита}

Рассматривается векторное преобразование Рисса $\nabla^{t} \Delta^{-(t+s) / 2} \operatorname{div}^{s}$ четного порядка $s+t$ в весовом пространстве $L_{2}\left(\mathbb{R}^{n} ;|x|^{a}\right)$. Устанавливается, что при $t \neq s$, $n>3$ его норма равна единице на некотором интервале значений $a$, и внутри интервала справедлива более силная оценка для подчиненной нормы.

Библиограффия: 3 названия.

Пусть $\mathbb{R}^{n}$ - евклидово пространство, $D=\left(D_{1}, \ldots, D_{n}\right)$ - оператор градиента $\left(D_{i}-\right.$ дифференцирование по координате $\left.x_{i}\right)$, div - coпряженньй к $D$ оператор, $\Delta^{-1}$ - оператор с символом $|\zeta|^{-2}$. Пусть $s, t$ - целые неотрицательные числа, $s+t$ - четное положительное число. Рассматривается векторное преобразование Рисса порядка $s+t-$ оператор

$$
T^{t, s}=D^{t} \Delta^{-(t+s) / 2} \operatorname{div}^{s},
$$

понимаемьй как оператор с символом $\left(\zeta_{i_{1}}, \ldots, \zeta_{i_{t}}|\zeta|^{-t-s} \zeta_{j_{1}}, \ldots, \zeta_{j_{s}}\right), i_{k}, j_{l}=1, \ldots, n$, и переводящий функции размерности $n^{s}$ в функции размерности $n^{t}$.

Вычисление или, по крайней мере, хорошие оценки норм классических операторов представляют значительный интерес ввиду их широкого распространения в приложениях. Традиционно рассматриваются преимущественно $L_{p}$-нормы (см., например, paботу [1] и список литературы в ней). В то же время известно, что при оценках в $L_{2}$ со степенным весом получение точных констант более вероятно, хотя в приложениях эти оценки обычно приводят к более слабым утверждениям, чем $L_{p}$-оценки. В данной работе рассматриваются $L_{2}$-оценки с весом $|x|^{a}$ для оператора $T^{t, s}$. При $a=0$ по равенству Парсеваля его норма, очевидно, равна единище. В работе устанавливается, что при $t \neq s, n>3$ норма равна единице на некотором отрезке, не исчерпьвающемся точкой $a=0$, и внутри отрезка справедлива более сильная оценка на некоторую подчиненную норму.

Отметим, что оператор $T^{t, s}$ естественно возникает при рассмотрении нелинейных эллиптических уравнений и систем высокого порядка [2], и результаты данной работы позволяют исследовать некоторьй класс вырождающихся нелинейных эллиптических уравнений и систем высокого порядка.

1. Основные результаты. Обозначим через $T_{a}^{t, s}$ норму оператора $T^{t, s}$ в пространстве $L_{2, a}=L_{2}\left(\mathbb{R}^{n} ;|x|^{a}\right)$ (строго говоря, из одной декартовой степени этого пространства в другую; показатели декартовой степени пространств будем для краткости опускать). 
Хорошо известно, что $T_{a}^{t, s}<\infty$ при $a \in(-n, n)$. Отметим следующие простые свойства:

1) $T_{0}^{t, s}=1$;

2) $T_{a}^{t, s} \geqslant 1$.

Действительно, при $a=0$ по равенству Парсеваля в $L_{2}$ без веса имеем $(\|\cdot\|$ обозначает норму в $L_{2}$ )

$$
\left\|T^{t, s} f\right\|=\left\|\zeta^{t}|\zeta|^{-t-s} \zeta^{s} \widehat{f}\right\| \leqslant\|\widehat{f}\|=\|f\|
$$

так что $T_{0}^{t, s} \leqslant 1$, но на функциях $f=D^{s} u$ при $u \in C_{0}^{\infty}$

$$
\left\|T^{t, s} f\right\|_{a}^{2}=\left\|D^{t} \Delta^{(s-t) / 2} u\right\|=\left\|D^{s} u\right\|=\|f\|,
$$

так что $T_{0}^{t, s}=1$.

Свойство 2) справедливо для практически любых весов. Пусть $\omega-$ вес в $\mathbb{R}^{n}$, который хотя бы в одной точке (в точке $\left.x_{0}\right)$ непрерывен и положителен. Пусть $B=B_{R}\left(x_{0}\right)$ - шар с центром $x_{0}$ радиуса $R$, и обозначим $\omega_{1}=\inf _{B} \omega(x), \omega_{2}=\sup _{B} \omega(x)$, где $R>0$ столь малое, что $\omega_{1}>0, \omega_{2}<\infty$. Выбирая $f=D^{s} u$ при $s \geqslant t$ и $f=D^{s} \Delta^{k} u, k=(t-s) / 2$, при $s<t$, где $u \in C_{0}^{\infty}(B)$, находим, что носитель $T^{t, s} f$ лежит в носителе $u$. Учитьвая (1), получаем $\left(\|\cdot\|_{\omega}\right.$ обозначает норму в $\left.L_{2}\left(\mathbb{R}^{n} ; \omega\right)\right)$

$$
\begin{aligned}
\left\|T^{t, s} f\right\|_{\omega} & =\left\|T^{t, s} f ; L_{2}(B ; \omega)\right\| \geqslant \omega_{1}^{1 / 2}\left\|T^{t, s} f ; L_{2}(B)\right\| \\
& =\omega_{1}^{1 / 2}\left\|T^{t, s} f\right\|=\omega_{1}^{1 / 2}\|f\| \geqslant\left(\frac{\omega_{1}}{\omega_{2}}\right)^{1 / 2}\|f\|_{\omega}
\end{aligned}
$$

По непрерьвности $\omega$ в точке $x_{0}$ величина $\omega_{1} / \omega_{2}$ может быть сделана произвольно близкой к единице при выборе достаточно малого $R>0$, что доказьвает оценку $T_{\omega}^{t, s} \geqslant 1$.

Обозначим $A=\left\{a \in(-n, n): T_{a}^{t, s}=1\right\}$. Поскольку $T_{0}^{t, s}=1, T_{a}^{t, s} \geqslant 1$ и $T_{a}^{t, s}$ логарифмически выпукло по $a$ по интерполящионной теореме Стейна-Вейса, это множество представляет собой отрезок (возможно, состоящий из одной точки $a=0$ ).

Обозначим $a_{*}=a_{*}(t, s, n)=\inf A, a^{*}=a^{*}(t, s, n)=\sup A$.

Пусть $\|\cdot\|_{a}$ - норма в $L_{2, a}, I_{1}$ - оператор с символом $|\zeta|^{-1}$ - потенциал Рисса порядка единица. Буквой $с$ будем обозначать различные несущественные положительные константы.

TEOPEMA 1. Пусть $n>3, t>s$. Тогда $a_{*}<0=a^{*} u$ npu $a \in\left(a_{*}, 0\right) \partial \Omega я f \in L_{2, a}$ виполнено

$$
\left\|T^{t, s} f\right\|_{a}^{2} \leqslant\|f\|_{a}^{2}-c a\left(a_{*}-a\right)\left\|I_{1} f\right\|_{a-2}^{2}
$$

константа $c>0$ зависит только от $t, s, n$. Кроме того, $a_{*} \geqslant 3-n$ и равенство $a_{*}=3-n$ достигается при $s=0$.

Tеорема 2. Пусть $n>3, s>t$. Тогдa $a^{*}>0=a_{*} u n p u a \in\left(0, a^{*}\right) \partial \Omega_{\text {g }} f \in L_{2, a}$ выполнено

$$
\left\|T^{t, s} f\right\|_{a}^{2} \leqslant\|f\|_{a}^{2}-c a\left(a^{*}-a\right)\left\|I_{1} f\right\|_{a-2}^{2}
$$


константа $c>0$ зависит только от $t, s, n$. Кроме того, $a^{*} \leqslant n-3$ и равенство $a^{*}=n-3$ достигается при $t=0$.

Отметим, что при сопряжении $\left(T^{t, s}\right)^{\prime}=T^{s, t},\left(L_{2, a}\right)^{\prime}=L_{2,-a}$, поэтому

$$
a^{*}(s, t, n)=-a_{*}(t, s, n)
$$

и для случая $s>t$ оценки границ интервала $a_{*}=0<a^{*} \leqslant n-3$, а также $a^{*}(0, s, n)=$ $n-3$ следуют из соответствующих оценок теоремы 1 . Однако, что касается оценки (3), она не следует из (2) по двойственности. А именно, при $t<s$ по двойственности и неравенству Юнга

$$
\left\|T^{t, s} f\right\|_{a}^{2}=\int f T^{s, t}\left(r^{a} T^{t, s} f\right) d x \leqslant \frac{1}{2}\left(\|f\|_{a}^{2}+\left\|T^{s, t}\left(r^{a} T^{t, s} f\right)\right\|_{-a}^{2}\right) .
$$

Применяя (2) при $-a \in\left(a_{*}(s, t, n), 0\right)$, получаем

$$
\left\|T^{t, s} f\right\|_{a}^{2} \leqslant\|f\|_{a}^{2}-c a\left(a^{*}(t, s, n)-a\right)\left\|I_{1}\left(r^{a} T^{t, s} f\right)\right\|_{-a-2}^{2} .
$$

Нетрудно проверить, что эта оценка следует из (3), но обратное неверно.

Укажем на возможность явной, хотя и неточной оценки сверху для $a_{*}$ при $t>s>0$. Она может быть получена из неравенства (7) в доказательстве леммы 1 при использовании явных выражений для норм $T_{a-2}^{2,0}, T_{a-2}^{1,1}$ (см. [2]). Из (7), очевидно, следует, что если $a \in(3-n, 0)$ удовлетворяет условию

$$
a \sum_{i=1}^{s}\left(T_{a-2}^{1,1}\right)^{2 i}+(a+n-3) \sum_{j=0}^{k-1}\left(T_{a-2}^{2,0}\right)^{-2 j} \geqslant 0
$$

то $a_{*} \leqslant a$, где $k=(t-s) / 2$. Если при $n>5$ ограничиться поисками оценки для $a_{*}$ на интервале $a \in(5-n, 0)$, то это выражение упрощается, так как $T_{a}^{2,0}=1$ при $3-n \leqslant a \leqslant 0$. Для $T^{1,1}$ при этом можно использовать простейшую оценку

$$
\left(T_{a}^{1,1}\right)^{2} \leqslant \frac{(n-2)^{2}}{(n-2)^{2}-a^{2}} .
$$

Поэтому если для $a \in(5-n, 0)$ вьполнено

$$
a \sum_{i=1}^{s}\left(\frac{(n-2)^{2}}{(n-a)(n+a-4)}\right)^{i}+(a+n-3) \frac{t-s}{2} \geqslant 0
$$

то $a_{*} \leqslant a$. Отсюда, в частности, следует, что при фиксированных $n, s$ всегда можно выбрать $t$ столь большое, что $a_{*}(t, s, n)<5-n$.

Отметим еще, что при $t>s>0$ показатель $a_{*}(t, s, n)$ не возрастает по $t$. Действительно, поскольку в любом случае $3-n \leqslant a_{*} \leqslant 0$, для $a \in(3-n, 0)$, учитьвая $T_{a}^{2,0}=1$, находим

$$
\left\|T^{t, s} f\right\|_{a}=\left\|T^{2,0}\left(T^{t-2, s} f\right)\right\|_{a} \leqslant\left\|T^{t-2, s} f\right\|_{a},
$$

так что $a_{*}(t, s, n) \leqslant a_{*}(t-2, s, n)$. 
2. Оценки операторов $T^{2,0}, T^{1,1}$. Установим для операторов $T^{2,0}$ и $T^{1,1}$ следующие оценки:

$$
\begin{gathered}
\left\|T^{2,0} f\right\|_{a}^{2}=\|f\|_{a}^{2}+a(a+n-3)\left\|D \Delta^{-1} f\right\|_{a-2}^{2}+a(2-a)\left\|\partial_{r} \Delta^{-1} f\right\|_{a-2}^{2}, \\
\left\|T^{1,1} f\right\|_{a}^{2} \leqslant\|f\|_{a}^{2}+a^{2}\left\|\Delta^{-1} \operatorname{div} f\right\|_{a-2}^{2},
\end{gathered}
$$

где $\partial_{r}$ - дифференцирование по $r=|x|, a \in(2-n, n), f \in L_{2, a}$.

Достаточно получить их для гладких финитных функций $f$, поскольку такие функции плотны в $L_{2, a}$. Пусть $\phi \in C_{0}^{\infty}$ - срезающая функция: $0 \leqslant \phi \leqslant 1, \phi=1$ в $B_{R}=\{x$ : $|x|<R\}, \phi=0$ вне $B_{2 R},\left|D^{j} \phi\right| \leqslant c_{j} R^{-j}$. Положим $\omega=r^{a} \phi$.

Для $T^{2,0}$, обозначая $u=\Delta^{-1} f$, интегрированием по частям находим

$$
\begin{aligned}
\left\|T^{2,0} f\right\|_{a}^{2}-\|f\|_{a}^{2} & =\int\left(\left|D^{2} u\right|^{2}-|\Delta u|^{2}\right) \omega d x=\int\left(-D_{i} D_{j} u D_{j} u D_{i} \omega+\Delta u D_{i} u D_{i} \omega\right) d x \\
& =\int\left(-2 D_{i} D_{j} u D_{j} u D_{i} \omega-D_{i} u D_{j} u D_{i} D_{j} \omega\right) d x \\
& =\int\left(|D u|^{2} \Delta \omega-D u D u D^{2} \omega\right) d x .
\end{aligned}
$$

Учитьвая явньй вид $\omega$ и вычисляя вторую производную от $r^{a}$

$$
D_{i} D_{j} r^{a}=a \delta_{i j} r^{a-2}+a(a-2) x_{i} x_{j} r^{a-4},
$$

находим

$$
\left\|T^{2,0} f\right\|_{\omega}^{2}-\|f\|_{\omega}^{2}=\int\left(a(a+n-3)|D u|^{2}+a(a-2)\left|\partial_{r} u\right|^{2}\right) r^{a-2} \phi d x+\Phi,
$$

где в $\Phi$ включены члены, содержащие производные $\phi$, которые, очевидно, удовлетворяют оценке

$$
|\Phi| \leqslant c R^{a-2} \int_{Q_{R}}|D u|^{2} d x ;
$$

здесь $Q_{R}=\{x: R<|x|<2 R\}$ - кольцо, содержащее носитель $D \phi$. При больших $R$ ввиду финитности $f$ имеем $|D u(x)| \leqslant c R^{1-n}$ в $Q_{R}$, поэтому при $R \rightarrow \infty$ получаем

$$
\left\|T^{2,0} f\right\|_{a}^{2}-\|f\|_{a}^{2}=a(a+n-3)\|D u\|_{a-2}^{2}+a(a-2)\left\|\partial_{r} u\right\|_{a-2}^{2}
$$

что доказьвает (4).

Для $T^{1,1}$ интегрированием по частям находим

$$
\begin{aligned}
\left\|T^{1,1} f\right\|_{\omega}^{2} & =\int\left|D \Delta^{-1} \operatorname{div} f\right|^{2} \omega d x=-\int \Delta^{-1} \operatorname{div} f\left(\omega \operatorname{div} f+D \omega D \Delta^{-1} \operatorname{div} f\right) d x \\
& =\int f\left(\omega D \Delta^{-1} \operatorname{div} f+D \omega \Delta^{-1} \operatorname{div} f\right) d x-\int \Delta^{-1} \operatorname{div} f D \omega D \Delta^{-1} \operatorname{div} f d x
\end{aligned}
$$


В первом интеграле по неравенству Юнга имеем (многоточием обозначено выражение, являющееся аргументом $f$ )

$$
\begin{aligned}
\left|\int f(\cdots) d x\right| \leqslant & \frac{1}{2}\|f\|_{\omega}^{2}+\frac{1}{2}\|(\cdots)\|_{1 / \omega}^{2} \\
= & \frac{1}{2}\|f\|_{\omega}^{2}+\frac{1}{2}\left(\left\|T^{1,1} f\right\|_{\omega}^{2}+2 \int D \Delta^{-1} \operatorname{div} f D \omega \Delta^{-1} \operatorname{div} f d x\right. \\
& \left.+\int\left|\Delta^{-1} \operatorname{div} f\right|^{2} \frac{|D \omega|^{2}}{\omega} d x\right),
\end{aligned}
$$

что приводит к оценке

$$
\left\|T^{1,1} f\right\|_{\omega}^{2} \leqslant\|f\|_{\omega}^{2}+\int\left|\Delta^{-1} \operatorname{div} f\right|^{2} \frac{|D \omega|^{2}}{\omega} d x
$$

Пусть $\phi=\phi_{1}^{2}$, где $\phi_{1}$ удовлетворяет тем же условиям, что и $\phi: \phi_{1} \in C_{0}^{\infty}\left(B_{2 R}\right), 0 \leqslant \phi_{1} \leqslant 1$, $\phi_{1}=1$ в $B_{R},\left|D \phi_{1}\right| \leqslant c / R$. Тогда

$\frac{|D \omega|^{2}}{\omega}=\frac{\left|\phi D r^{a}+r^{a} D \phi\right|^{2}}{r^{a} \phi}=a^{2} r^{a-2} \phi+2 a r^{a-1} \partial_{r} \phi+4 r^{a}\left|D \phi_{1}\right|^{2} \leqslant a^{2} r^{a-2} \phi+c R^{a-2} \chi_{R}$,

где $\chi_{R}$ - характеристическая функция кольца $Q_{R}$, содержащего носитель $D \phi$. Это дает

$$
\left\|T^{1,1} f\right\|_{\omega}^{2} \leqslant\|f\|_{\omega}^{2}+a^{2}\left\|r^{-1} \Delta^{-1} \operatorname{div} f\right\|_{\omega}^{2}+c R^{a-2}\left\|\Delta^{-1} \operatorname{div} f ; L_{2}\left(Q_{R}\right)\right\|^{2} .
$$

При больших $R$ ввиду финитности $f$ имеем $\left|\Delta^{-1} \operatorname{div} f(x)\right| \leqslant c R^{1-n}$ в $Q_{R}$, поэтому при $R \rightarrow \infty$ отсюда следует (5).

3. Оценка нормы для малых $а$. Здесь мы, учитьвая, что оператор $T^{t, s}$ при $t>s, t+s$ четном, является тензорным произведением тензорных степеней операторов $T^{2,0}, T^{1,1}$, на основании оценок $(4),(5)$ установим следующий результат.

ЛЕмма 1. Пусть $t>s, n>3$. Существует $b<0$ такое, что при $a \in(b, 0)$ для $f \in L_{2, a}$ выполнено

$$
\left\|T^{t, s} f\right\|_{a}^{2} \leqslant\|f\|_{a}^{2}+c a\left\|D \Delta^{-1} f\right\|_{a-2}^{2}
$$

әде с $>0$ зависит только от $s, t, n$. При $s=0$ можкно взять $b=3-n, c=a+n-3$.

ДокАЗАТЕЛЬСтво. Пусть $s=0$. Обозначая $k=t / 2$, имеем $T^{t, s}=T^{2 k, 0}=\left(T^{2,0}\right)^{k}$ и по (4) находим

$$
\begin{aligned}
\left\|T^{t, s} f\right\|_{a}^{2}= & \left\|\left(T^{2,0}\right)^{k-1} f\right\|_{a}^{2}+a(a+n-3)\left\|D \Delta^{-1}\left(T^{2,0}\right)^{k-1} f\right\|_{a-2}^{2} \\
& +a(2-a)\left\|\partial_{r} \Delta^{-1}\left(T^{2,0}\right)^{k-1} f\right\|_{a-2}^{2}=\cdots \\
= & \|f\|_{a-2}^{2}+\sum_{i=1}^{k}\left(a(a+n-3)\left\|D \Delta^{-1}\left(T^{2,0}\right)^{k-i} f\right\|_{a-2}^{2}\right. \\
& \left.+a(2-a)\left\|\partial_{r} \Delta^{-1}\left(T^{2,0}\right)^{k-i} f\right\|_{a-2}^{2}\right) .
\end{aligned}
$$


При $a \in(3-n, 0)$, поскольку в сумме по $i$ все коэффициенты отрицательны, отсюда следует оценка

$$
\left\|T^{t, s} f\right\|_{a}^{2} \leqslant\|f\|_{a}^{2}+a(a+n-3)\left\|D \Delta^{-1} f\right\|_{a-2}^{2}
$$

что доказьвает лемму для $s=0$.

Рассмотрим случай $s>0$. Обозначая $g=T^{t-s, 0} f$, по (5) находим

$$
\begin{aligned}
\left\|T^{t, s} f\right\|_{a}^{2} & =\left\|\left(T^{1,1}\right)^{s} g\right\|_{a}^{2} \leqslant\left\|\left(T^{1,1}\right)^{s-1} g\right\|_{a}^{2}+a^{2}\left\|\Delta^{-1} \operatorname{div}\left(T^{1,1}\right)^{s-1} g\right\|_{a-2}^{2} \leqslant \cdots \\
& \leqslant\|g\|_{a}^{2}+a^{2} \sum_{i=1}^{s}\left\|\Delta^{-1} \operatorname{div}\left(T^{1,1}\right)^{s-i} g\right\|_{a-2}^{2} .
\end{aligned}
$$

Для нормы $g$ по (4) при $a<0$, обозначая $k=(t-s) / 2$, имеем

$$
\begin{aligned}
\|g\|_{a}^{2} & =\left\|\left(T^{2,0}\right)^{k} f\right\|_{a}^{2} \leqslant\left\|\left(T^{2,0}\right)^{k-1} f\right\|_{a}^{2}+a(a+n-3)\left\|D \Delta^{-1}\left(T^{2,0}\right)^{k-1} f\right\|_{a-2}^{2} \leqslant \cdots \\
& \leqslant\|f\|_{a}^{2}+a(a+n-3) \sum_{j=1}^{k}\left\|D \Delta^{-1}\left(T^{2,0}\right)^{k-j} f\right\|_{a-2}^{2} .
\end{aligned}
$$

Отметим, что $\Delta^{-1} \operatorname{div} T^{2,0}=T^{1,1} D \Delta^{-1}$. Теперь для нормы $T^{t, s}$ получаем

$$
\begin{aligned}
\left\|T^{t, s} f\right\|_{a}^{2} \leqslant & \|f\|_{a}^{2}+a(a+n-3) \sum_{j=1}^{k}\left\|D \Delta^{-1}\left(T^{2,0}\right)^{k-j} f\right\|_{a-2}^{2} \\
& +a^{2} \sum_{i=0}^{s-1}\left\|\left(T^{1,1}\right)^{s-i} D \Delta^{-1}\left(T^{2,0}\right)^{k-1} f\right\|_{a-2}^{2} .
\end{aligned}
$$

Оператор $T^{1,1}$, являясь композицией проекторов Рисса, ограничен в $L_{2, b}$ при $b \in(-n, n)$, и его норма ограничена равномерно по $b$ на любом внутреннем подьнтервале интервала $(-n, n)$. Поэтому

$$
\left\|\left(T^{1,1}\right)^{s-i} D \Delta^{-1}\left(T^{2,0}\right)^{k-1} f\right\|_{a-2} \leqslant c\left\|D \Delta^{-1}\left(T^{2,0}\right)^{k-1} f\right\|_{a-2} ;
$$

здесь константа $c$ может быть выбрана не зависящей от $a \in(3-n, 0)$. Это дает $\left\|T^{t, s} f\right\|_{a}^{2} \leqslant\|f\|_{a}^{2}+a(a+n-3) \sum_{j=1}^{k}\left\|D \Delta^{-1}\left(T^{2,0}\right)^{k-j} f\right\|_{a-2}^{2}+c a^{2}\left\|D \Delta^{-1}\left(T^{2,0}\right)^{k-1} f\right\|_{a-2}^{2}$.

При $a \rightarrow-0$ коэффициент в последнем слагаемом равен $O\left(a^{2}\right)$, а во втором слагаемом равен $-O(|a|)$, поэтому на достаточно малом интервале $a \in(b, 0)$ отсюда следует оценка (6).

4. Оценка нормы для $a$, удаленных от нуля. На интервале $a \in\left(a_{*}, b\right)$ оценка нормы оператора $T^{t, s}$ устанавливается с помощью интерполяции. Мы следуем доказательству теоремы Рисса-Торина с одинаковыми показателями и различньми весами, как оно изложено в [3, теорема 5.4.1], но при этом учитываем младшие члены. 
Tеорема 3. Пусть $a_{0}, a_{1} \in(2-n, n)$, линейный оператор $T: L_{2, a_{i}} \rightarrow L_{2, a_{i}}$, $i=0,1$, удовлетворяет оценкам

$$
\begin{gathered}
\|T f\|_{a_{0}} \leqslant\|f\|_{a_{0}}, \\
\|T f\|_{a_{1}}^{2} \leqslant\|f\|_{a_{1}}^{2}-c_{1}\left\|D \Delta^{-1} f\right\|_{a_{1}-2}^{2},
\end{gathered}
$$

где $c_{1}>0$. Тогда при $a \in\left(a_{0}, a_{1}\right)$ для $f \in L_{2, a}$ выполнено

$$
\|T f\|_{a}^{2} \leqslant\|f\|_{a}^{2}-c\left|a-a_{0}\right| \cdot\left\|D \Delta^{-1} f\right\|_{a-2}^{2},
$$

әде $c>0$.

ДокАЗАТЕЛЬСтво. Пусть $\omega_{0}(x), \omega_{1}(x)$ - два неотрицательных веса в $\mathbb{R}^{n}$, и положим

$$
K(\rho, f)=\inf _{f_{0}+f_{1}=f}\left(\left\|f_{0}\right\|_{\omega_{0}}^{2}+\rho^{2}\left\|f_{1}\right\|_{\omega_{1}}^{2}\right),
$$

где $\|\cdot\|_{\omega}$ обозначает норму в $L_{2}\left(\mathbb{R}^{n} ; \omega\right)$. Обозначая

$$
\varphi(\rho)=\inf _{y_{0}+y_{1}=1}\left(\left|y_{0}\right|^{2}+\rho\left|y_{1}\right|^{2}\right),
$$

имеем

$$
K(\rho, f)=\int_{\mathbb{R}^{n}} \inf _{f_{0}+f_{1}=f}\left(\left|f_{0}\right|^{2} \omega_{0}+\rho^{2}\left|f_{1}\right|^{2} \omega_{1}\right) d x=\int_{\mathbb{R}^{n}}|f|^{2} \omega_{0} \varphi\left(\rho^{2} \frac{\omega_{1}}{\omega_{0}}\right) d x .
$$

При $\theta \in(0,1)$

$$
\begin{aligned}
\int_{0}^{\infty} \rho^{-2 \theta} K(\rho, f) \frac{d \rho}{\rho} & =\int_{0}^{\infty} \rho^{-2 \theta} \int_{\mathbb{R}^{n}}|f|^{2} \omega_{0} \varphi\left(\rho^{2} \frac{\omega_{1}}{\omega_{0}}\right) d x \frac{d \rho}{\rho} \\
& =\int_{\mathbb{R}^{n}}|f|^{2} \omega_{0} \int_{0}^{\infty} \rho^{-2 \theta} \varphi\left(\rho^{2} \frac{\omega_{1}}{\omega_{0}}\right) \frac{d \rho}{\rho} d x \\
& =\int_{\mathbb{R}^{n}}|f|^{2} \omega_{0}^{1-\theta} \omega_{1}^{\theta}\left(\int_{0}^{\infty}\left(\rho^{2} \frac{\omega_{1}}{\omega_{0}}\right)^{-\theta} \varphi\left(\rho^{2} \frac{\omega_{1}}{\omega_{0}}\right) \frac{d \rho}{\rho}\right) d x=c(\theta)\|f\|_{\omega}^{2},
\end{aligned}
$$

где

$$
c(\theta)=\int_{0}^{\infty} \rho^{-2 \theta} \varphi\left(\rho^{2}\right) \frac{d \rho}{\rho}<\infty
$$

с учетом $\varphi(\rho)<\min (1, \rho)$, и мы обозначили $\omega=\omega_{0}^{1-\theta} \omega_{1}^{\theta}$.

Для $a \in\left(a_{0}, a_{1}\right)$ обозначим $\theta=\left(a-a_{0}\right) /\left(a_{1}-a_{0}\right) \in(0,1), \omega=r^{a}, \omega_{0}=r^{a_{0}}, \omega_{1}=r^{a_{1}}$. По доказанному выше при $f \in L_{2, \omega}$ для нормы $f$ имеем представление

$$
\|f\|_{\omega}^{2}=\frac{1}{c(\theta)} \int_{0}^{\infty} \rho^{-2 \theta} \inf _{f_{0}+f_{1}=f}\left(\left\|f_{0}\right\|_{\omega_{0}}^{2}+\rho^{2}\left\|f_{1}\right\|_{\omega_{1}}^{2}\right) \frac{d \rho}{\rho} .
$$

Обозначим через $f_{0, \rho}+f_{1, \rho}=f$ то разбиение $f$, для которого достигается равенство

$$
\left|f_{0, \rho}\right|^{2} \omega_{0}+\rho^{2}\left|f_{1, \rho}\right|^{2} \omega_{1}=\inf _{f_{0}+f_{1}=f}\left(\left|f_{0}\right|^{2} \omega_{0}+\rho^{2}\left|f_{1}\right|^{2} \omega_{1}\right) .
$$


Ввиду линейности $T$ для $g=T f$ имеем $g=T f_{0, \rho}+T f_{1, \rho}$ при каждом $\rho$. Заменяя оптимальное разбиение $g$ разбиением $T f_{0, \rho}+T f_{1, \rho}$ и используя оценки $(8),(9)$, получаем

$$
\begin{aligned}
c(\theta)\|g\|_{\omega}^{2} & =\int_{0}^{\infty} \rho^{-2 \theta} \inf _{g_{0}+g_{1}=g}\left(\left\|g_{0}\right\|_{\omega_{0}}^{2}+\rho^{2}\left\|g_{1}\right\|_{\omega_{1}}^{2}\right) \frac{d \rho}{\rho} \\
& \leqslant \int_{0}^{\infty} \rho^{-2 \theta}\left(\left\|T f_{0, \rho}\right\|_{\omega_{0}}^{2}+\rho^{2}\left\|T f_{1, \rho}\right\|_{\omega_{1}}^{2}\right) \frac{d \rho}{\rho} \\
& \leqslant \int_{0}^{\infty} \rho^{-2 \theta}\left(\left\|f_{0, \rho}\right\|_{\omega_{0}}^{2}+\rho^{2}\left\|f_{1, \rho}\right\|_{\omega_{1}}^{2}-\rho^{2} c_{1}\left\|r^{-1} D \Delta^{-1} f_{1, \rho}\right\|_{\omega_{1}}^{2}\right) \frac{d \rho}{\rho} \\
& =c(\theta)\|f\|_{\omega}^{2}-c \int_{0}^{\infty} \rho^{2-2 \theta}\left\|r^{-1} D \Delta^{-1} f_{1, \rho}\right\|_{\omega_{1}}^{2} \frac{d \rho}{\rho} .
\end{aligned}
$$

Теперь достаточно показать, что

$$
\left\|r^{-1} D \Delta^{-1} f\right\|_{\omega}^{2} \leqslant c \int_{0}^{\infty}\left\|r^{-1} D \Delta^{-1} f_{1, \rho}\right\|_{\omega_{1}}^{2} \frac{d \rho}{\rho} .
$$

В (10) при исследовании на минимум с помощью дифференцирования легко устанавливается, что

$$
f_{1, \rho}=\frac{f}{1+\rho^{2} \omega_{1} / \omega_{0}} .
$$

Обозначая $F_{1, \rho}=D \Delta^{-1} f_{1, \rho}$, имеем

$$
\begin{aligned}
D \Delta^{-1} f & =D \Delta^{-1}\left(\left(1+\rho^{2} \frac{\omega_{1}}{\omega_{0}}\right) \operatorname{div} F_{1, \rho}\right) \\
& =D \Delta^{-1} \operatorname{div}\left(\left(1+\rho^{2} \frac{\omega_{1}}{\omega_{0}}\right) F_{1, \rho}\right)-D \Delta^{-1}\left(F_{1, \rho} \rho^{2} D \frac{\omega_{1}}{\omega_{0}}\right) .
\end{aligned}
$$

Обозначая $\sigma=\sigma_{\rho}=r^{-2} \omega_{1} /\left(1+\rho^{2} \omega_{1} / \omega_{0}\right)^{2}$, получаем

$$
\begin{aligned}
\left\|D \Delta^{-1} f\right\|_{\sigma} & \leqslant\left\|D \Delta^{-1} \operatorname{div}\left(\left(1+\rho^{2} \frac{\omega_{1}}{\omega_{0}}\right) F_{1, \rho}\right)\right\|_{\sigma}+\left\|D \Delta^{-1}\left(F_{1, \rho} \rho^{2} D \frac{\omega_{1}}{\omega_{0}}\right)\right\|_{\sigma} \\
& \equiv\left\|D \Delta^{-1} \operatorname{div} g_{1}\right\|_{\sigma}+\left\|D \Delta^{-1} g_{2}\right\|_{\sigma} .
\end{aligned}
$$

Временно введем дополнительное предположение $a_{1}+2\left(a_{0}-a_{1}\right) \in(2-n, n)$. Во втором слагаемом, заменяя $\sigma$ большим весом $r^{-2} \omega_{0}^{2} /\left(\rho^{4} \omega_{1}\right)$ и учитьвая, что $2 a_{0}-a_{1}-2>$ $-n$, по неравенству Харди получаем

$$
\left\|D \Delta^{-1} g_{2}\right\|_{\sigma} \leqslant \frac{c}{\rho^{2}}\left\|D^{2} \Delta^{-1} g_{2}\right\|_{\omega_{0}^{2} / \omega_{1}} .
$$

Поскольку $2 a_{0}-a_{1} \in(-n, n)$, оператор $D^{2} \Delta^{-1}$, являясь композицией проекторов Рисса, ограничен в $L_{2}$ с весом $\omega_{0}^{2} / \omega_{1}$, что дает

$$
\left\|D \Delta^{-1} g_{2}\right\|_{\sigma} \leqslant \frac{c}{\rho^{2}}\left\|g_{2}\right\|_{\omega_{0}^{2} / \omega_{1}} \leqslant c\left\|r^{-1} F_{1, \rho}\right\|_{\omega_{1}} .
$$


В первом слагаемом в $(12)$ для $1 / \sigma_{\rho}$ имеем

$$
\frac{1}{\sigma_{\rho}}=\frac{r^{2}}{\omega_{1}}+2 \rho^{2} \frac{r^{2}}{\omega_{0}}+\rho^{4} \frac{r^{2} \omega_{1}}{\omega_{0}^{2}} \equiv \nu_{1}+\nu_{2}+\nu_{3}
$$

Поскольку $2-a_{1}, 2-a_{0}, a_{1}+2-2 a_{0} \in(-n, n)$, оператор $D \Delta^{-1} \operatorname{div}$, являясь композицией проекторов Рисса, ограничен в $L_{2, \nu}, i=1,2,3$, множители $\rho^{2}, \rho^{4}$ не влияют на величину норм. Значит, он ограничен в $L_{2,1 / \sigma}$ и его норма

$$
\left\|D \Delta^{-1} \operatorname{div}\right\|_{1 / \sigma} \leqslant \max _{i=1,2,3}\left\|D \Delta^{-1} \operatorname{div}\right\|_{\nu_{i}}
$$

оценивается величиной, не зависящей от $\rho \in(0, \infty)$. Так как при сопряжении

$$
\left(D \Delta^{-1} \operatorname{div}\right)^{\prime}=D \Delta^{-1} \operatorname{div}, \quad\left(L_{2,1 / \sigma}\right)^{\prime}=L_{2, \sigma},
$$

для его нормы в $L_{2, \sigma}$ справедлива та же оценка. Поэтому

$$
\left\|D \Delta^{-1} \operatorname{div} g_{1}\right\|_{\sigma} \leqslant c\left\|g_{1}\right\|_{\sigma}=c\left\|r^{-1} F_{1, \rho}\right\|_{\omega_{1}} .
$$

Из (12) получаем

$$
\left\|D \Delta^{-1} f\right\|_{\sigma} \leqslant c\left\|r^{-1} D \Delta^{-1} f_{1, \rho}\right\|_{\omega_{1}}
$$

и, следовательно,

$$
\begin{aligned}
c \int_{0}^{\infty} & \rho^{2-2 \theta}\left\|r^{-1} D \Delta^{-1} f_{1, \rho}\right\|_{\omega_{1}}^{2} \frac{d \rho}{\rho} \\
& \geqslant \int_{0}^{\infty} \rho^{2-2 \theta}\left\|D \Delta^{-1} f\right\|_{\sigma}^{2} \frac{d \rho}{\rho}=\int_{0}^{\infty} \rho^{2-2 \theta} \int_{\mathbb{R}^{n}}\left|D \Delta^{-1} f\right|^{2} \frac{r^{-2} \omega_{1}}{\left(1+\rho^{2} \omega_{1} / \omega_{0}\right)^{2}} d x \frac{d \rho}{\rho} \\
& =\int_{\mathbb{R}^{n}}\left|D \Delta^{-1} f\right|^{2} r^{-2} \omega_{0}^{1-\theta} \omega_{1}^{\theta}\left(\int_{0}^{\infty} \frac{\left(\rho^{2} \omega_{1} / \omega_{0}\right)^{1-\theta}}{\left(1+\rho^{2} \omega_{1} / \omega_{0}\right)^{2}} \frac{d \rho}{\rho}\right) d x=c_{1}(\theta)\left\|r^{-1} D \Delta^{-1} f\right\|_{\omega}^{2},
\end{aligned}
$$

где

$$
c_{1}(\theta)=\int_{0}^{\infty} \frac{\rho^{2-2 \theta}}{\left(1+\rho^{2}\right)^{2}} \frac{d \rho}{\rho}>\frac{1}{4} \int_{0}^{1} \rho^{2-2 \theta} \frac{d \rho}{\rho}=\frac{1}{8(1-\theta)} .
$$

Поскольку для $c(\theta)$ из $\varphi(\rho)<\min (1, \rho)$ следует $c(\theta)<1 /(2 \theta(1-\theta))$, из (11) получаем

$$
\|T f\|_{\omega}^{2} \leqslant\|f\|_{\omega}^{2}-\theta c\left\|r^{-1} D \Delta^{-1} f\right\|_{\omega}^{2} .
$$

Если $a_{1}+2\left(a_{0}-a_{1}\right) \in(2-n, n)$, это завершает доказательство теоремы. Если же нет, то (пусть для определенности $a_{0}<a_{1}$ ) выберем $b>0$ такое, что $a_{0}-2 b>2-n$. По интерполяционной теореме Стейна-Вейса норма $T$ в $L_{2, a}$ не превосходит единицы при всех $a \in\left(a_{0}, a_{1}\right)$. Предположения теоремы вместе с добавочным предположением $a_{1}+2\left(a_{0}-a_{1}\right) \in(2-n, n)$ вьполнены при замене $a_{0}$ на $a_{0}^{\prime}=a_{1}-2 b$, поэтому утверждение теоремы справедливо для $a \in\left(a_{0}^{\prime}, a_{1}\right)$; в частности,

$$
\|T f\|_{a}^{2} \leqslant\|f\|_{a}^{2}-c\left\|D \Delta^{-1} f\right\|_{a-2}^{2}
$$

при $a=a_{1}-b$ c некоторой константой $c>0$. Теперь (если $\left.a_{0}<a_{1}-3 b\right)$ предположения теоремы вместе с добавочным предположением выполнены на концах интервала $\left(a_{1}-3 b, a_{1}-b\right)$ и, следовательно, (13) вьполнено при $a=a_{1}-2 b$. После конечного числа итераций получаем утверждение теоремы для всего интервала $\left(a_{0}, a_{1}\right)$. 
5. Оценка границ интервала. Здесь мы на основании равенства (4) установим следующее утверждение.

Лемма 2. Пусть $t>s, n \geqslant 3$. Тогда $a^{*}=0, a_{*} \geqslant 3-n$.

ДоказАтЕльство. Докажем $a^{*}=0$. Возьмем $f=D^{s} \Delta^{k} u, k=(t-s) / 2$, где $u \in C_{0}^{\infty}$. На функциях $g=D^{s} v$ оператор $T^{s, s}$ действует как единичньй:

$$
T^{s, s} g=D^{s} \Delta^{-s} \operatorname{div}^{s}\left(D^{s} v\right)=D^{s} \Delta^{-s} \Delta^{s} v=D^{s} v=g
$$

Поэтому

$$
T^{t, s} f=T^{t-s, 0}\left(T^{s, s} f\right)=T^{t-s, 0} f=\left(T^{2,0}\right)^{k} f
$$

и по равенству (4) при $a \in(0,2)$, отбрасьвая последнее неотрицательное слагаемое, получаем

$$
\begin{aligned}
\left\|T^{t, s} f\right\|_{a}^{2} & =\left\|\left(T^{2,0}\right)^{k} f\right\|_{a}^{2} \geqslant\left\|\left(T^{2,0}\right)^{k-1} f\right\|_{a}^{2}+a(a+n-3)\left\|D \Delta^{-1}\left(T^{2,0}\right)^{k-1} f\right\|_{a-2}^{2} \geqslant \cdots \\
& \geqslant\|f\|_{a}^{2}+a(a+n-3) \sum_{i=1}^{k}\left\|D \Delta^{-1}\left(T^{2,0}\right)^{k-i} f\right\|_{a-2}^{2} \\
& =\|f\|_{a}^{2}+a(a+n-3) \sum_{i=0}^{k-1}\left\|D^{t-1-2 i} \Delta^{i} u\right\|_{a-2}^{2}
\end{aligned}
$$

Ограничимся слагаемым с $i=0$, которое присутствует для любого $k \geqslant 1$, т.е. при любом $t>s$ (с учетом условия четности $t+s)$. Для финитной функции $u$, не являющейся тождественным нулем, норма $D^{t-1} u$ больше нуля. Поэтому $T_{a}^{t, s}>1$ при $a>0$ и, следовательно, $a^{*} \leqslant 0$. Но по равенству Парсеваля в $L_{2}$ без веса $T_{0}^{t, s}=1$, так что $a^{*}=0$.

Докажем $a_{*} \geqslant 3-n$. Пусть $\psi_{1}, \psi_{2}-$ гладкие функции на $\mathbb{R}$ с носителями на интервалах $(-1,1)$ и $(1,2)$ соответственно. Положим $g(x)=\psi_{1}\left(x_{1}\right), \phi(x)=\psi_{2}\left(\left|x^{\prime}\right| / R\right)$, где $x^{\prime}=\left(x_{2}, \ldots, x_{n}\right), R>1$ - параметр. Положим $u=g \phi, f=D^{s} \Delta^{k} u, k=(t-s) / 2$. Тогда, как и при доказательстве $a^{*}=0$,

$$
T^{t, s} f=\left(T^{2,0}\right)^{k} f
$$

и по равенству (4)

$$
\begin{aligned}
\left\|T^{t, s} f\right\|_{a}^{2}= & \left\|\left(T^{2,0}\right)^{k} f\right\|_{a}^{2} \\
= & \|f\|_{a}^{2}+\sum_{i=1}^{k}\left(a(a+n-3)\left\|D \Delta^{-1}\left(T^{2,0}\right)^{k-i} f\right\|_{a-2}^{2}\right. \\
& \left.+a(2-a)\left\|\partial_{r} \Delta^{-1}\left(T^{2,0}\right)^{k-i} f\right\|_{a-2}^{2}\right) \\
= & \|f\|_{a}^{2}+\sum_{i=0}^{k-1}\left(a(a+n-3)\left\|D^{t-1-2 i} \Delta^{i} u\right\|_{a-2}^{2}\right. \\
& \left.+a(2-a)\left\|\partial_{r} D^{t-2-2 i} \Delta^{i} u\right\|_{a-2}^{2}\right) .
\end{aligned}
$$


При $a<3-n$ для коэффициентов имеем $a(a+n-3)>0, a(2-a)<0$. Рассмотрим, как будут вести себя нормы в сумме по $i$ с ростом $R$. Для норм при положительном коэффициенте $a(a+n-3)$ ограничимся слагаемым с $i=0$, которое всегда присутствует при $t>s$. При $i=0$ имеем

$$
\left\|D^{t-1} u\right\|_{a-2}^{2} \geqslant\left\|D_{1}^{t-1} u\right\|_{a-2}^{2}=\left\|\phi D_{1}^{t-1} g\right\|_{a-2}^{2}
$$

Обозначим через $G_{R}=\left\{x:\left|x_{1}\right|<1, R<\left|x^{\prime}\right|<2 R\right\}$ множество, содержащее носитель $u$. Тогда

$$
\left\|\phi D_{1}^{t-1} g\right\|_{a-2}^{2} \geqslant \inf _{G_{R}} r^{a-2} \int_{\mathbb{R}}\left|D_{1}^{t-1} g\right|^{2} d x_{1} \int_{\mathbb{R}^{n-1}}|\phi|^{2} d x^{\prime} \geqslant c_{0} R^{a+n-3}
$$

с некоторой константой $c_{0}>0$.

Нормы при коэффициенте $a(2-a)$ оценим сверху. Имеем

$$
\left|\partial_{r} D^{t-2-2 i} \Delta^{i} u\right| \leqslant c\left|\phi \partial_{r} D_{1}^{t-2} g\right|+c \sum_{j=1}^{t-1}\left|D^{j} \phi\right| \cdot\left|D_{1}^{t-1-j} g\right|
$$

В первом слагаемом

$$
\partial_{r} D_{1}^{t-2} g=\frac{x_{1}}{r} D_{1}^{t-1} g
$$

и, поскольку $\left|x_{1}\right|<1$ на носителе $u$, находим

$$
\left\|\phi \partial_{r} D_{1}^{t-2} g\right\|_{a-2}^{2} \leqslant\left\|\phi D_{1}^{t-1} g\right\|_{a-4}^{2} \leqslant c\left|G_{R}\right| \sup _{G_{R}} r^{a-4} \leqslant c R^{a+n-5} .
$$

Для слагаемых, содержащих производные $\phi$, учитьвая $\left|D^{j} \phi\right| \leqslant c_{j} R^{-j}$, получаем

$$
\sum_{j=1}^{t-1}\left\|D^{j} \phi D_{1}^{t-1-j} g\right\|_{a-2}^{2} \leqslant c R^{-2}\left|G_{R}\right| \sup _{G_{R}} r^{a-2} \leqslant c R^{a+n-5}
$$

В (14) это дает при $a<3-n$

$$
\left\|T^{t, s} f\right\|_{a}^{2} \geqslant\|f\|_{a}^{2}+a(a+n-3) c_{0} R^{a+n-3}+a(2-a) c R^{a+n-5} .
$$

Выбирая $R$ столь большое, что

$$
(3-n-a) c_{0}>(2-a) c R^{-2}
$$

получаем

$$
\left\|T^{t, s} f\right\|_{a}^{2}>\|f\|_{a}^{2}
$$

откуда следует $T_{a}^{t, s}>1$. Поскольку неравенство установлено при произвольном $a<$ $3-n$, это дает $a_{*} \geqslant 3-n$. 
6. Доказательство теоремы 1. По лемме $2 a_{*} \geqslant 3-n, a^{*}=0$, а по лемме 1 $a_{*}<0, a_{*}(t, 0, n) \leqslant 3-n$.

Из леммы 1 и теоремы 3 с учетом того, что $a_{*} \geqslant 3-n$, следует оценка

$$
\left\|T^{t, s} f\right\|_{a}^{2} \leqslant\|f\|_{a}^{2}-c a\left(a_{*}-a\right)\left\|D \Delta^{-1} f\right\|_{a-2}^{2}
$$

на интервале $a \in\left(a_{*}, 0\right)$. Поскольку $I_{1} f=I_{1} \operatorname{div}\left(D \Delta^{-1} f\right)$ и оператор $I_{1} \operatorname{div}$ ограничен в $L_{2, b}$ равномерно по $b$ на каждом внутреннем подьнтервале интервала $(-n, n)$, имеем

$$
\left\|I_{1} f\right\|_{a-2} \leqslant c\left\|D \Delta^{-1} f\right\|_{a-2}
$$

с константой $c$, не зависящей от $a \in\left(a_{*}, 0\right)$, что влечет оценку $(2)$.

7. Оценка оператора $T^{0,2}$. Установим для оператора $T^{0,2}$ при $n>3$ оценку

$$
\left\|T^{0,2} f\right\|_{a}^{2} \leqslant\|f\|_{a}^{2}-c a\left\|I_{1} f\right\|_{a-2}^{2}
$$

для $f \in L_{2, a}$ на малом интервале $a \in(0, b), b>0$, где $b, c$ зависят только от $n$.

Используем декомпозицию Ходжа второго порядка для функций размерности $n^{2}$, на которых действует $T^{0,2}$ :

$$
f=D^{2} u+D v_{1}+D v_{2}+w
$$

в том смысле, что для $f=\left(f_{i j}\right), i, j=1, \ldots, n$,

$$
f_{i j}=D_{i} D_{j} u+D_{j} v_{1 i}+D_{i} v_{2 j}+w_{i j}
$$

где $\operatorname{div} v_{1}=0, \operatorname{div} v_{2}=0$ и $\operatorname{div} w=0$ в обоих вариантах: $D_{i} w_{i j}=0, D_{j} w_{i j}=0$, по повторяющимся индексам подразумевается суммирование от 1 до $n$. Существование такой декомпозиции легко усмотреть: для $f=\left(f_{i j}\right) \in L_{2, a}, a \in(-n, n)$, достаточно положить $u=\Delta^{-2} \operatorname{div}^{2} f, v_{1 i}=\Delta^{-1} D_{j} f_{i j}-D_{i} u, v_{2 j}=\Delta^{-1} D_{i} f_{i j}-D_{j} u$. Тогда, очевидно, $D^{2} u, D v_{1}, D v_{2}, w \in L_{2, a}$.

Имеем

$$
T^{0,2} f=\Delta^{-1} \operatorname{div}^{2}\left(D^{2} u+D v_{1}+D v_{2}+w\right)=\Delta u
$$

и по (4)

$$
\left\|T^{0,2} f\right\|_{a}^{2}=\|\Delta u\|_{a}^{2}=\left\|D^{2} u\right\|_{a}^{2}-a(a+n-3)\|D u\|_{a-2}^{2}-a(2-a)\left\|\partial_{r} u\right\|_{a-2}^{2} .
$$

Для нормы $f$ имеем

$$
\begin{aligned}
\|f\|_{a}^{2}= & \left\|D^{2} u\right\|_{a}^{2}+\left\|D v_{1}\right\|_{a}^{2}+\left\|D v_{2}\right\|_{a}^{2}+\|w\|_{a}^{2} \\
& +2 \int\left(D^{2} u D v_{1}+D^{2} u D v_{2}+D^{2} u w+D v_{1} D v_{2}+D v_{1} w+D v_{2} w\right) r^{a} d x
\end{aligned}
$$

так что при $a>0$

$$
\|f\|_{a}^{2}-\left\|T^{0,2} f\right\|_{a}^{2} \geqslant a(n-3)\|D u\|_{a-2}^{2}+\left\|D v_{1}\right\|_{a}^{2}+\left\|D v_{2}\right\|_{a}^{2}+\|w\|_{a}^{2}+2 \int \cdots .
$$


При интегрировании по частям с учетом равенства нулю дивергенций $v_{1}, v_{2}, w$ находим

$$
2 \int \cdots=-2 \int\left(D u D v_{1}+D u D v_{2}+D u w+\frac{1}{2}\left(v_{1} D v_{2}+D v_{1} v_{2}\right)+v_{1} w+v_{2} w\right) D r^{a} d x
$$

Учитьвая, что $\left|D r^{a}\right|=a r^{a-1}$, по неравенству Юнга получаем

$$
\begin{aligned}
\left|2 \int \ldots\right| \leqslant & 3 a^{3 / 2}\|D u\|_{a-2}^{2}+\frac{3}{2} a^{1 / 2}\left(\left\|D v_{1}\right\|_{a}^{2}+\left\|D v_{2}\right\|_{a}^{2}\right) \\
& +\frac{3}{2} a^{3 / 2}\left(\left\|v_{1}\right\|_{a-2}^{2}+\left\|v_{2}\right\|_{a-2}^{2}\right)+3 a^{1 / 2}\|w\|_{a}^{2}
\end{aligned}
$$

По неравенству Харди

$$
\|D v\|_{a} \geqslant \frac{a+n-2}{2}\|v\|_{a-2}
$$

так что при $a \in(0,1)$

$$
\left|2 \int \cdots\right| \leqslant 3 a^{3 / 2}\|D u\|_{a-2}^{2}+3 a^{1 / 2}\left(\left\|D v_{1}\right\|_{a}^{2}+\left\|D v_{2}\right\|_{a}^{2}+\|w\|_{a}^{2}\right) .
$$

Из (16) получаем

$$
\|f\|_{a}^{2}-\left\|T^{0,2} f\right\|_{a}^{2} \geqslant\left(1-3 a^{1 / 2}\right)\left(a\|D u\|_{a-2}^{2}+\left\|D v_{1}\right\|_{a}^{2}+\left\|D v_{2}\right\|_{a}^{2}+\|w\|_{a}^{2}\right)
$$

Для $I_{1} f$ имеем

$$
\left\|I_{1} f\right\|_{a-2} \leqslant\left\|I_{1} D^{2} u\right\|_{a-2}+\left\|I_{1} D v_{1}\right\|_{a-2}+\left\|I_{1} D v_{2}\right\|_{a-2}+\left\|I_{1} w\right\|_{a-2} .
$$

В трех последних слагаемых применим неравенство Харди

$$
\frac{a+n-2}{2}\left\|I_{1} z\right\|_{a-2} \leqslant\left\|D I_{1} z\right\|_{a}
$$

и в силу ограниченности оператора $D I_{1}$ в $L_{2, b}, b \in(-n, n)$, получаем

$$
\left\|I_{1} f\right\|_{a-2}^{2} \leqslant c\left(\|D u\|_{a-2}+\left\|D v_{1}\right\|_{a}+\left\|D v_{2}\right\|_{a}+\|w\|_{a}\right)
$$

Теперь из (17) для достаточно малого $b>0$ при $a \in(0, b)$ следует $(15)$.

8. Доказательство теоремы 2. Утверждения теоремы 2 о величине $a_{*}, a^{*}$ следуют из теоремы 1 по двойственности.

На малом интервале $a \in(0, b)$ оценка (3) устанавливается на основании оценок (15), (5) аналогично доказательству леммы 1 . Обозначая $g=T^{0, s-t} f$, по (5) находим

$$
\left\|T^{t, s} f\right\|_{a}^{2}=\left\|\left(T^{1,1}\right)^{t} g\right\|_{a}^{2} \leqslant\|g\|_{a}^{2}+a^{2} \sum_{i=1}^{t}\left\|\Delta^{-1} \operatorname{div}\left(T^{1,1}\right)^{t-i} g\right\|_{a-2}^{2}
$$


и далее, поскольку проекторы Рисса ограничены в $L_{2, a-2}$ равномерно по $a \in\left(0, a^{*}\right)$,

$$
\left\|T^{t, s} f\right\|_{a}^{2} \leqslant\|g\|_{a}^{2}+c a^{2}\left\|I_{1} g\right\|_{a-2}^{2}
$$

Для нормы $g$ в силу (15) при $a \in(0, b)$, обозначая $k=(s-t) / 2$, имеем

$$
\|g\|_{a}^{2}=\left\|\left(T^{0,2}\right)^{k} f\right\|_{a}^{2} \leqslant\|f\|_{a}^{2}-c_{1} a \sum_{j=1}^{k}\left\|I_{1}\left(T^{0,2}\right)^{k-j} f\right\|_{a-2}^{2}
$$

Теперь для нормы $T^{t, s}$ получаем

$$
\left\|T^{t, s} f\right\|_{a}^{2} \leqslant\|f\|_{a}^{2}-c_{1} a \sum_{j=1}^{k}\left\|I_{1}\left(T^{0,2}\right)^{k-j} f\right\|_{a-2}^{2}+c a^{2}\left\|I_{1}\left(T^{0,2}\right)^{k} f\right\|_{a-2}^{2}
$$

откуда следует $(3)$ для $a \in(0, b)$ при достаточно малом $b>0$ (вообще говоря, гораздо меньшем, чем в (15)).

На интервале $a \in\left(b, a^{*}\right)$ оценка (3) следует из теоремы 3 , поскольку для $a_{0}=a^{*}$ имеем $T_{a_{0}}^{t, s}=1$, а для $a_{1}=b$ установлено (9) (отметим, что операторы $I_{1}$ и $D \Delta^{-1}$, очевидно, оценивают друг друга в $L_{2, a-2}$ при $\left.a \in(2-n, n+2)\right)$ и оба числа $a_{0}, a_{1}$ лежат в интервале $(2-n, n)$.

\section{СПИСОК ЦИТИРОВАННОЙ ЛИТЕРАТУРЫ}

[1] Iwaniec T., Gaven M. Riesz transforms and related singular integrals // J. Reine Angew. Math. 1996. V. 473. P. 25-59.

[2] Калита Е. А. Разрешимость нелинейных эллиптических систем в пространствах слабее естественного энергетического // Изв. РАН. Сер. матем. 1997. Т. 61. № 2. С. 53-80.

[3] Берг Й. , Лёфстрём Й. Интерполяционные пространства. Введение. М.: Мир, 1980.

Институт прикладной математики и механики

НАН Украины, г. Донецк

Поступило

E-mail: kalita@iamm.ac.donetsk.ua 